\title{
Characteristics of Milk Fermented by Streptococcus thermophilus MGA45-4 and the Profiles of Associated Volatile Compounds during Fermentation and Storage
}

\author{
Tong Dan, Rulin Jin, Weiyi Ren, Ting Li, Haiyan Chen and Tiansong Sun * \\ Key Laboratory of Dairy Biotechnology and Engineering, Ministry of Education, Inner Mongolia Agricultural \\ University, 306 Zhaowuda Street, Hohhot 010018, China; dantong813218@imau.edu.cn (T.D.); \\ jrl_happy@163.com (R.J.); renweiyi153@163.com (W.R.); 18848114353@163.com (T.L.); \\ m15024943239@163.com (H.C.) \\ * Correspondence: sts9940@sina.com; Tel.: +86-471-431-9940; Fax: +86-471-430-0122
}

Received: 9 March 2018; Accepted: 9 April 2018; Published: 11 April 2018

\begin{abstract}
The lactic acid bacterium Streptococcus thermophilus is a major starter culture for the production of dairy products. In this study, the physiochemical characteristics of milk fermented by the MGA45-4 isolate of $S$. thermophilus were analyzed. Our data indicate that milk fermented using S. thermophilus MGA45-4 maintained a high viable cell count (8.86 $\log 10$ colony-forming units $/ \mathrm{mL}$ ), and a relatively high $\mathrm{pH}(4.4)$, viscosity (834.33 $\mathrm{mPa} \cdot \mathrm{s})$, and water holding capacity $(40.85 \%)$ during 14 days of storage. By analyzing the volatile compound profile using solid-phase microextraction and gas chromatography/mass spectrometry, we identified 73 volatile compounds in the fermented milk product, including five carboxylic acids, 21 aldehydes, 13 ketones, 16 alcohols, five esters, and 13 aromatic carbohydrates. According to the odor activity values, 11 of these volatile compounds were found to play a key role in producing the characteristic flavor of fermented milk, particularly octanal, nonanal, hexanal, 2,3-butanedione, and 1-octen-3-ol, which had the highest odor activity values among all compounds analyzed. These findings thus provide more insights in the chemical/molecular characteristics of milk fermented using $S$. thermophilus, which may provide a basis for improving dairy product flavor/odor during the process of fermentation and storage.
\end{abstract}

Keywords: Streptococcus thermophilus; fermented milk; fermentation characteristics; solid-phase microextraction (SPME); gas chromatography/mass spectrometry (GC-MS)

\section{Introduction}

Streptococcus thermophilus is a Gram-positive, non-pathogenic, facultative anaerobic lactic acid bacterium (LAB). This bacterium is related to other LABs, such as Lactococcus lactis, which is the most important industrial starter culture widely used in the dairy industry [1]. Among the opportunistic bacterial pathogens that are used for the preparation of dairy products, S. thermophilus is the only species generally recognized as being safe [2]. This species can be used alone or in combination with other LABs for the production of dairy products such as cheese [3,4], yogurt [5] and fermented camel milk [6]. Therefore, $S$. thermophilus has been proven to have industrial value [2].

Streptococcus thermophilus has the ability to metabolize lactose into exopolysaccharides, vitamins, and several flavor compounds [7]. In dairy products, multiple categories of volatile organic aroma compounds have been identified including carboxylic acids, aldehydes, ketones, alcohols, and esters [8-11]. These volatile compounds provide the texture, mouthfeel, and taste/odor of dairy products and therefore they are the key determining factors of product quality [12-15]. Although many volatile compounds have been identified, only a few, such as acetic acid, are considered to have a significant influence on the final flavor of dairy products. Acetic acid can be produced during 
storage at low temperatures and it is the major component responsible for increasing the acidic flavor of many dairy products [16]. Recent studies have demonstrated that acetic acid is frequently found in fermented milk [16], fermented camel milk [6], and in goat cheese [8].

To better elucidate how much each volatile compound contributes to the overall flavor of a sample, the odor activity value (OAV) has been developed and applied to the product evaluation process $[17,18]$. OAVs are represented as the ratios between the concentration of each compound and its detection threshold concentration [19]. If the OAV of a flavor compound is greater than 1, the compound is defined as an active odorant. In a recent study, several compounds with an OAV greater than one were identified, which may have an important impact on the aroma of fermented cow milk [20].

The main advantages of solid-phase microextraction (SPME) as a pretreatment method are its simplicity, low cost, ease of automation, and solvent-free extraction [16]. SPME combined with gas chromatography-mass spectrometry (SPME-GC-MS) has been used to study the volatile composition of a wide range of products [20].

Industrial strains of LAB can be isolated from different milk environments, including fermented milk, cheese, and kefir [21]. In China, yogurt has become a major fermented dairy product. However, few studies have examined the profile of volatile compounds in milk fermented with LAB strains isolated from traditional dairy products. Therefore, this study investigated aroma formation and the fermentation characteristics of milk that had been fermented by S. thermophilus MGA45-4 from traditional fermented milk collected in Kent Province, Mongolia and tested its behavior as a starter in the yogurt industry [22].

\section{Results and Discussion}

\subsection{Physicochemical Characteristics of Milk Fermented Using S. thermophilus}

To evaluate the fermentation characteristics of $S$. thermophilus MGA45-4, we measured the viable cell counts, $\mathrm{pH}, \mathrm{TA}, \mathrm{WHC}$, and viscosity of fermented milk samples. The trends for changes in viable cell counts differed between fermentation and storage (Figure 1A). Specifically, while the viable cell count increased gradually during fermentation, it peaked at the 12th day during storage $\left(9.1688 \log _{10}\right.$ $\mathrm{CFU} / \mathrm{mL}$ ), followed by a steady decline (Figure 1A). The $\mathrm{pH}$ of samples decreased from 6.63 to 4.5 within the first $6.5 \mathrm{~h}$ of fermentation (Figure 1B). This value remained almost unchanged (4.4) during the 14 days of storage, suggesting that carboxylic acid production by $S$. thermophilus MGA45-4 is inhibited at a refrigeration temperature of $4{ }^{\circ} \mathrm{C}$. Unlike $\mathrm{pH}$, the TA value increased steadily during fermentation, reaching $75.72{ }^{\circ} \mathrm{T}$ at the final stage of the process (Figure 1C). A continuous but smaller increase was observed in TA during storage, with the value changing from $75.72{ }^{\circ} \mathrm{T}$ on the first day to $95.07^{\circ} \mathrm{T}$ on the 14 th day of storage (Figure 1C). During the fermentation period, the viscosity of fermented milk increased significantly over time and a maximum value of $834.33 \mathrm{mPa} \cdot \mathrm{s}$ was reached on day 1 of storage (Figure 1D). Overall, the fermented milk had a significantly higher viscosity in storage than during fermentation. Consistent with this, milk samples in storage also had a markedly higher water-holding capacity (WHC) compared with those in the fermentation process (Figure 1E). On the third day of storage, the WHC reached a maximum value of $48.01 \%$, which was significantly greater than the WHC obtained from the other time points (Figure 1E). Sensory assessment indicated that products stored for $0 \mathrm{~d}$ and $12 \mathrm{~h}$ at $4{ }^{\circ} \mathrm{C}$ were better than the other samples.

\subsection{Analysis of Volatile Compounds in Fermented Milk}

Odor/flavor formation in dairy products is a complex process which involves glycolysis, lipolysis, and proteolysis of various chemical components. Depending on the starter culture isolates used for fermentation, the final quality of fermented milk can vary significantly. To evaluate the aroma profile of milk fermented by S. thermophilus MGA45-4, we used the SPME-GC/MS technique to analyze the volatile compound composition of samples during fermentation and storage. As shown in Table 1, 
we identified 73 compounds based on the retention indexes (RIs) calculated using the HP-5MS column. These compounds included various types of carboxylic acids, aldehydes, ketones, alcohols, esters, and aromatic carbohydrates. The range of the RI for almost all compounds was \pm 5 or \pm 10 units.
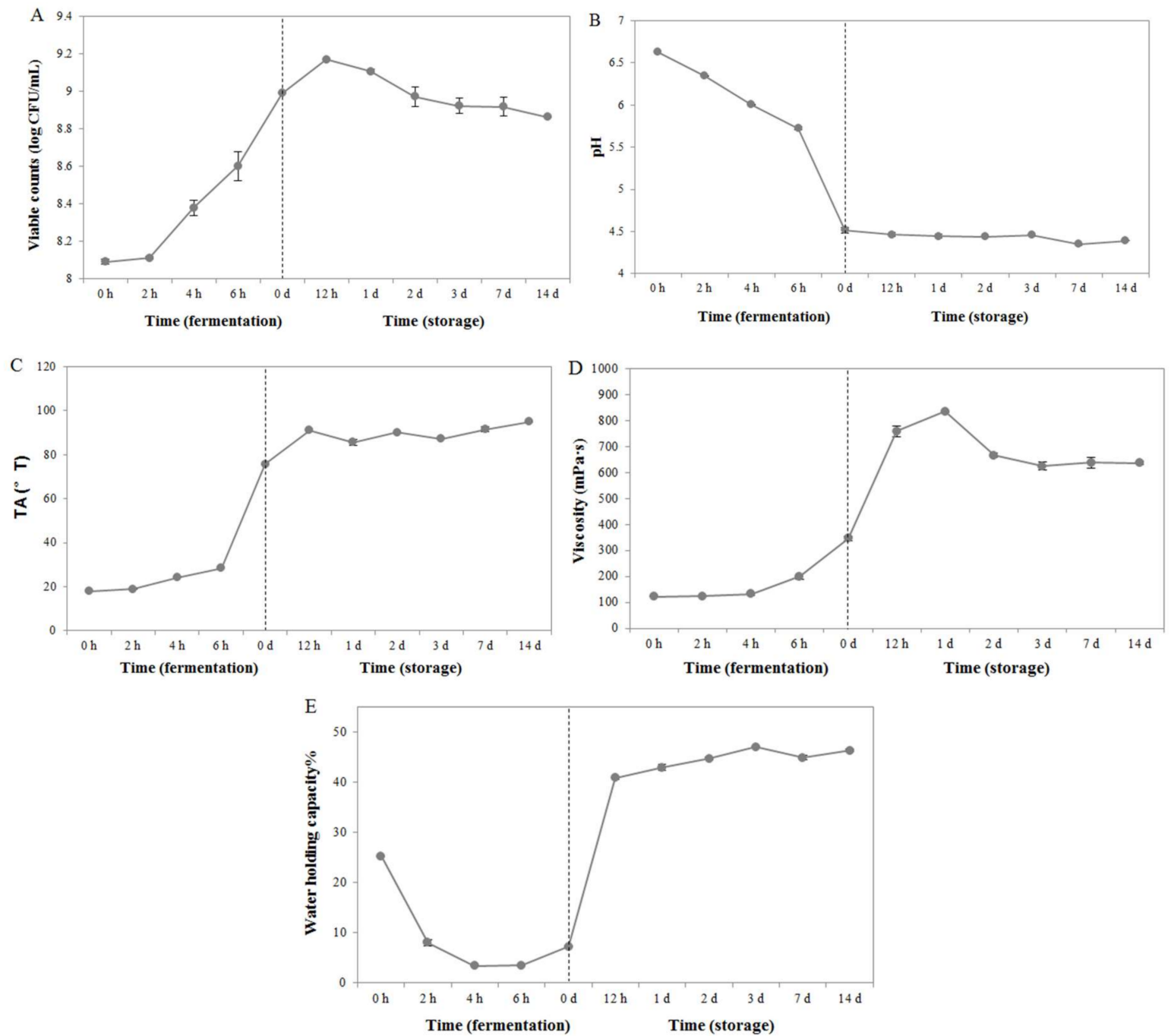

Figure 1. The physiochemical characteristics of milk fermented by $S$. thermophilus MGA45-4 during fermentation $(0 \mathrm{~h}, 2 \mathrm{~h}, 4 \mathrm{~h}$, and $6 \mathrm{~h})$ and storage $(0 \mathrm{~d}, 12 \mathrm{~h}, 1 \mathrm{~d}, 2 \mathrm{~d}, 3 \mathrm{~d}, 7 \mathrm{~d}$, and $14 \mathrm{~d})$. The parameters analyzed included (A) the count of viable $S$. thermophilus MGA45-4 cells, (B) pH, (C) titratable acidity, (D) viscosity, and (E) water-holding capacity of the fermented milk samples.

Acids are the precursors of methyl ketones, alcohols, lactones, and esters; as such, they are important for the generation of odors in dairy products [23]. Using the SPME pretreatment method combined with GC/MS, we identified five different carboxylic acids from the $S$. thermophilus MGA45-4 fermented milk (Table 1). During the storage period, two short-chain fatty acids, acetic acid and hexanoic acid, were detected, which ranged in concentration from 17.21-70.07 $\mu \mathrm{g} / \mathrm{L}$, and 7.36-35.17 $\mu \mathrm{g} / \mathrm{L}$, respectively. Given their strong odor, short-chain fatty acids $(\mathrm{C}<6)$ are particularly crucial for odor generation in fermented milk compared with other acid compounds [24]. Previous studies have demonstrated that acetic acid is responsible for the vinegary, pungent, acidic odor associated with dairy products [25], while hexanoic acid has a sickening, sweet, rancid, cheese-like odor [26]. Two additional acids, nonanoic and octanoic acids, were found in samples from the storage process, but were absent from those undergoing fermentation (Table 1). In particular, the concentration 
of octanoic acid reached as high as $3.64 \mu \mathrm{g} / \mathrm{L}$ on the second day of storage, which was consistent with earlier findings reported by Condurso et al. [8].

Based on the SPME/GC/MS analysis, we identified 21 types of aldehydes in the volatile compound profile (Table 1). Aldehyde compounds are short-lived constituents of dairy products as they are rapidly converted into the corresponding alcohols or acids such as 3-methylbutanal and hexanal upon production $[27,28]$. As shown in Table 1, high levels of 3-methyl-butanal $(7.52-16.95 \mu \mathrm{g} / \mathrm{L})$ were detected in milk samples during fermentation. This is in agreement with the earlier finding that 3-methylbutanal is an important determinant of odor in dairy products [29]. We also detected a significant amount of benzaldehyde $(5.65-7.14 \mu \mathrm{g} / \mathrm{L})$ in samples undergoing fermentation. Benzaldehyde is produced from phenylacetaldehyde through $\alpha$-oxidation or from cinnamic acid through $\beta$-oxidation [30]. This compound is also frequently found in dairy products such as cheese [8], fermented milk [18], and fermented camel milk [6].

The predominant group of volatile compounds found in dairy products is ketones, such as 2,3-butanedione which can be produced from methyl-ketones through $\alpha$-oxidation of fatty acids [31]. In this study, we identified 13 ketone compounds in samples from the fermentation and storage stages. Based on their concentrations, the main ketone compounds included 2,3-butanedione (9.03-15.25 $\mu \mathrm{g} / \mathrm{L})$, acetoin (3.22-58.48 $\mu \mathrm{g} / \mathrm{L})$, 2-heptanone (17.43-53.88 $\mu \mathrm{g} / \mathrm{L})$, and 2-nonanone (5.95-32.78 $\mu \mathrm{g} / \mathrm{L})$. 2,3-Butanedione and acetoin were present in both the fermentation and storage stages, except at the end of storage. Acetoin can be produced from 2,3-butanedione as a byproduct of LAB metabolism, and both of these compounds can be further converted into other metabolites such as 2-butanone, 2-butanol, and butane-2,3-diol [32]. Our data were similar to those of an earlier report which indicated that 2,3-butanedione and acetoin can be detected in fermented milk and may be important for the final determination of product odor [11].

In addition to ketones, the type and amount of alcohol compounds produced can also have a significant impact on the aroma of dairy products. Some alcohol compounds can be derived from amino acids or aldehydes. For example, 3-methyl-butanol is generated through the reduction of the corresponding aldehydes [33]. In our study, 17 types of alcohol compounds were identified in the aroma profile of fermented milk (Table 1). Based on their concentrations, the main alcohol compounds

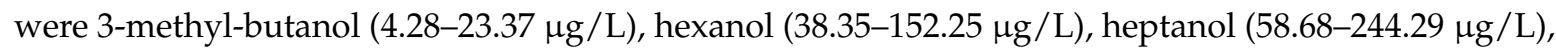
and 1-nonanol (12.11-45.23 $\mu \mathrm{g} / \mathrm{L})$. 1-Octen-3-ol (1.09-9.88 $\mu \mathrm{g} / \mathrm{L})$ was detected in samples undergoing fermentation and during storage. This compound produces a powerful, sweet, and earthy odor and, therefore, it may play a key role in generating the characteristic flavor of fermented milk [34].

Ester compounds are another group of volatile constituents that influence the odor outcome of dairy products [35]. The substrates required for the biosynthesis of ester compounds are mostly generated through the metabolism of carbohydrates and fat as well as catabolism of amino acids [36]. In the volatile fraction of samples analyzed, we identified five ester compounds and the main ester types included acetic acid octyl ester (1.23-7.83 $\mu \mathrm{g} / \mathrm{L})$, formic acid octyl ester (32.29-132.2 $\mu \mathrm{g} / \mathrm{L})$, and formic acid hexyl ester (1.01-2.66 $\mu \mathrm{g} / \mathrm{L}$ ) (Table 1). Esters contribute to the generation of fruity odors such as apple-like, pear-like, and banana-like odors [37]. Due to their relatively low detection threshold values (e.g., the threshold value of acetic acid octyl ester is $12 \mu \mathrm{g} / \mathrm{L}$ ) [38], they can have a significant impact on the aroma of dairy products.

Aromatic carbohydrates can be characterized directly by GC-MS because the degradation process of these compounds is relatively slow [39]. Using GC-MS, we identified 13 aromatic hydrocarbon compounds in milk fermented by $S$. thermophilus MGA45-4. Those with a relatively high concentration were heptane (2.21-8.38 $\mu \mathrm{g} / \mathrm{L})$, 1-octene (3.94-5.91 $\mu \mathrm{g} / \mathrm{L})$, and 1-nonene (5.48-46.1 $\mu \mathrm{g} / \mathrm{L})$, which were consistent with previously reported findings [16]. 
Table 1. Volatile compounds produced in milk fermented by S. thermophilus MGA45-4 during fermentation and storage.

\begin{tabular}{|c|c|c|c|c|c|c|c|c|c|c|c|c|c|c|c|c|c|}
\hline & \multirow{2}{*}{ Volatile Compound } & \multirow{2}{*}{$\begin{array}{l}\text { Chemical } \\
\text { Formula }\end{array}$} & \multirow{2}{*}{$\mathrm{RT}^{1}$} & \multirow[b]{2}{*}{$\mathrm{RI}^{2}$} & \multirow{2}{*}{$\mathrm{RI}^{3}$} & \multirow{2}{*}{ Method $^{4}$} & \multicolumn{11}{|c|}{$\mu \mathrm{g} / \mathrm{L}$} \\
\hline & & & & & & & $0 \mathrm{~h}(\mathrm{~F})^{5}$ & $2 \mathrm{~h} \mathrm{(F)}$ & $4 \mathrm{~h} \mathrm{(F)}$ & $6 \mathrm{~h} \mathrm{(F)}$ & $0 \mathrm{~d}(\mathrm{~S})^{6}$ & $12 \mathrm{~h}(\mathrm{~S})$ & $1 \mathrm{~d}(\mathrm{~S})$ & $2 d(S)$ & $3 \mathrm{~d}(\mathrm{~S})$ & $7 \mathrm{~d}(\mathrm{~S})$ & $14 \mathrm{~d}(\mathrm{~S})$ \\
\hline \multicolumn{18}{|c|}{ Carboxylic acid compounds } \\
\hline 1 & Acetic acid & $\mathrm{C}_{2} \mathrm{H}_{4} \mathrm{O}_{2}$ & 2.71 & 630.71 & 638 & MS, RI & - & - & - & - & 70.07 & 24.76 & 21.08 & 24.28 & 19.80 & 18.19 & 17.21 \\
\hline 2 & Hexanoic acid & $\mathrm{C}_{6} \mathrm{H}_{12} \mathrm{O}_{2}$ & 14.72 & 1021.01 & 1020 & MS, RI & - & - & - & - & 35.17 & 29.77 & 7.68 & 7.49 & 7.89 & 7.52 & 7.36 \\
\hline 3 & Heptanoic acid & $\mathrm{C}_{7} \mathrm{H}_{14} \mathrm{O}_{2}$ & 15.80 & 1053.46 & 1062 & MS, RI & - & - & - & - & 8.31 & 4.34 & 3.05 & 3.47 & 3.33 & 2.15 & 3.20 \\
\hline 4 & Nonanoic acid & $\mathrm{C}_{9} \mathrm{H}_{18} \mathrm{O}_{2}$ & 21.09 & 1224.43 & 1226 & MS, RI & - & - & - & - & - & - & - & 0.75 & 0.18 & - & - \\
\hline 5 & Octanoic acid & $\mathrm{C}_{8} \mathrm{H}_{16} \mathrm{O}_{2}$ & 20.21 & 1193.85 & 1191 & MS, RI & - & - & - & - & - & - & - & 3.64 & - & - & - \\
\hline \multicolumn{18}{|c|}{ Aldehydes } \\
\hline 1 & Methacrolein & $\mathrm{C}_{4} \mathrm{H}_{6} \mathrm{O}$ & 2.30 & 599.69 & 567 & MS, RI & - & - & - & 1.38 & - & - & - & - & - & - & - \\
\hline 2 & Butanal, 3-methyl- & $\mathrm{C}_{5} \mathrm{H}_{10} \mathrm{O}$ & 3.47 & 689.08 & 689 & MS, RI & 7.52 & 15.02 & 16.95 & 12.62 & - & - & - & - & - & - & - \\
\hline 3 & Butanal, 3-hydroxy- & $\mathrm{C}_{4} \mathrm{H}_{8} \mathrm{O}_{2}$ & 3.53 & 693.79 & - & MS & - & - & - & - & 4.45 & 6.83 & 3.59 & 1.79 & - & - & - \\
\hline 4 & 2-Pentenal, (E)- & $\mathrm{C}_{5} \mathrm{H}_{8} \mathrm{O}$ & 5.52 & 765.69 & 765 & MS, RI & - & - & - & 1.93 & 1.15 & 1.03 & 1.02 & 1.23 & 1.25 & 1.60 & 1.88 \\
\hline 5 & Hexanal & $\mathrm{C}_{6} \mathrm{H}_{12} \mathrm{O}$ & 6.87 & 809.39 & 809 & MS, RI & 99.08 & 91.58 & 58.12 & 52.03 & 3.96 & - & - & - & - & - & - \\
\hline 6 & 2-Pentenal, 2-methyl- & $\mathrm{C}_{6} \mathrm{H}_{10} \mathrm{O}$ & 7.53 & 826.57 & 826 & MS, RI & - & - & - & - & 1.89 & - & - & - & - & - & - \\
\hline 7 & 2-Hexenal, (E)- & $\mathrm{C}_{6} \mathrm{H}_{10} \mathrm{O}$ & 8.95 & 863.25 & 861 & MS, RI & 1.91 & 1.92 & 1.96 & 5.58 & 6.33 & 2.82 & 1.89 & 2.03 & 1.71 & 1.71 & 1.93 \\
\hline 8 & Heptanal & $\mathrm{C}_{7} \mathrm{H}_{14} \mathrm{O}$ & 10.87 & 905.14 & 906 & MS, RI & 191.95 & 129.22 & 78.60 & 32.93 & 4.41 & 1.84 & 1.01 & 1.25 & 1.73 & 1.34 & 2.25 \\
\hline 9 & 2-Heptenal, (E)- & $\mathrm{C}_{7} \mathrm{H}_{12} \mathrm{O}$ & 12.87 & 965.33 & 964 & MS, RI & 10.03 & 11.41 & 10.42 & 11.53 & 23.47 & 7.45 & 3.39 & 6.20 & 2.97 & 4.90 & 5.78 \\
\hline 10 & Benzaldehyde & $\mathrm{C}_{7} \mathrm{H}_{6} \mathrm{O}$ & 12.94 & 970.87 & 971 & MS, RI & 7.14 & 6.81 & 5.65 & - & - & - & - & - & - & - & - \\
\hline 11 & Octanal & $\mathrm{C}_{8} \mathrm{H}_{16} \mathrm{O}$ & 14.57 & 1009.21 & 1005 & MS, RI & 89.55 & 45.12 & 23.49 & 18.24 & 7.73 & 2.95 & - & - & - & - & - \\
\hline 12 & 2,4-Heptadienal, (E,E)- & $\mathrm{C}_{7} \mathrm{H}_{10} \mathrm{O}$ & 14.81 & 1023.54 & 1023 & MS, RI & - & 1.42 & 1.85 & 1.40 & 1.15 & - & - & - & - & - & - \\
\hline 13 & 2-Octenal, (E)- & $\mathrm{C}_{8} \mathrm{H}_{14} \mathrm{O}$ & 16.41 & 1069.45 & 1067 & MS, RI & 13.29 & 13.86 & 15.07 & 15.66 & 20.48 & 10.21 & 4.74 & 4.44 & 3.58 & 3.49 & 4.28 \\
\hline 14 & Nonanal & $\mathrm{C}_{9} \mathrm{H}_{18} \mathrm{O}$ & 17.92 & 1118.91 & 1119 & MS, RI & 52.04 & 35.95 & 28.60 & 9.58 & 4.01 & 1.52 & 1.43 & 1.03 & 1.50 & 1.12 & 1.13 \\
\hline 15 & 2-Nonenal, (E)- & $\mathrm{C}_{9} \mathrm{H}_{16} \mathrm{O}$ & 19.62 & 1172.50 & 1172 & MS, RI & 26.20 & 30.44 & 22.48 & 25.42 & 31.14 & 18.42 & 6.26 & 12.15 & 4.62 & 6.46 & 6.58 \\
\hline 16 & Decanal & $\mathrm{C}_{10} \mathrm{H}_{20} \mathrm{O}$ & 21.02 & 1216.62 & 1214 & MS, RI & 3.63 & 2.83 & 2.59 & 1.87 & - & - & - & - & - & - & - \\
\hline 17 & 2-Decenal, (Z)- & $\mathrm{C}_{10} \mathrm{H}_{18} \mathrm{O}$ & 22.61 & 1277.90 & 1280 & MS, RI & 12.13 & 15.01 & 19.53 & 22.75 & 28.22 & 16.54 & 7.93 & 7.64 & 7.42 & 6.07 & 7.02 \\
\hline 18 & 2-Decenal, (E)- & $\mathrm{C}_{10} \mathrm{H}_{18} \mathrm{O}$ & 22.75 & 1281.57 & 1279 & MS, RI & 3.80 & 6.69 & 19.01 & - & - & - & - & - & - & - & - \\
\hline 19 & Undecanal & $\mathrm{C}_{11} \mathrm{H}_{22} \mathrm{O}$ & 23.51 & 1310.47 & 1300 & MS, RI & - & - & - & - & 1.94 & - & - & - & - & - & - \\
\hline 20 & 2-Undecenal, E- & $\mathrm{C}_{11} \mathrm{H}_{20} \mathrm{O}$ & 24.55 & 1349.47 & 1340.00 & MS, RI & - & - & - & 1.56 & - & - & - & - & - & - & - \\
\hline 21 & 2-Undecenal & $\mathrm{C}_{11} \mathrm{H}_{20} \mathrm{O}$ & 24.81 & 1359.26 & 1359 & MS, RI & 2.12 & 3.50 & 6.48 & 6.25 & 8.91 & 4.28 & 3.65 & 3.53 & 2.44 & 2.02 & 2.22 \\
\hline \multicolumn{18}{|c|}{ Ketones } \\
\hline 1 & 2,3-Butanedione & $\mathrm{C}_{4} \mathrm{H}_{6} \mathrm{O}_{2}$ & 2.16 & STD & MS. STD & MS, RI & - & - & - & - & 16.08 & 16.49 & 15.25 & 16.07 & 9.03 & - & - \\
\hline 2 & 2-Butanone, 3-methyl- & $\mathrm{C}_{5} \mathrm{H}_{10} \mathrm{O}$ & 2.26 & STD & MS. STD & MS, RI & - & 3.34 & 5.89 & 18.97 & 16.91 & 16.13 & 10.85 & 10.25 & 8.54 & 7.84 & 7.01 \\
\hline 3 & 2-Pentanone & $\mathrm{C}_{5} \mathrm{H}_{10} \mathrm{O}$ & 3.42 & 685.33 & 687 & MS, RI & - & - & - & - & - & - & - & 0.78 & - & - & - \\
\hline
\end{tabular}


Table 1. Cont

\begin{tabular}{|c|c|c|c|c|c|c|c|c|c|c|c|c|c|c|c|c|c|}
\hline & \multirow{2}{*}{ Volatile Compound } & \multirow{2}{*}{$\begin{array}{l}\text { Chemical } \\
\text { Formula }\end{array}$} & \multirow[b]{2}{*}{$\mathrm{RT}^{1}$} & \multirow[b]{2}{*}{$\mathrm{RI}^{2}$} & \multirow[b]{2}{*}{$\mathrm{RI}^{3}$} & \multirow[b]{2}{*}{ Method $^{4}$} & \multicolumn{11}{|c|}{$\mu \mathrm{g} / \mathrm{L}$} \\
\hline & & & & & & & $0 \mathrm{~h}(\mathrm{~F})^{5}$ & $2 \mathrm{~h}(\mathrm{~F})$ & $4 \mathrm{~h}(\mathrm{~F})$ & $6 \mathrm{~h} \mathrm{(F)}$ & $0 \mathrm{~d}(\mathrm{~S})^{6}$ & $12 \mathrm{~h}(\mathrm{~S})$ & $1 \mathrm{~d}(\mathrm{~S})$ & $2 \mathrm{~d}(\mathrm{~S})$ & $3 \mathrm{~d}(\mathrm{~S})$ & $7 \mathrm{~d}(\mathrm{~S})$ & $14 \mathrm{~d}(\mathrm{~S})$ \\
\hline 4 & Acetoin & $\mathrm{C}_{4} \mathrm{H}_{8} \mathrm{O}_{2}$ & 4.22 & 720.87 & MS. STD & MS & - & - & 3.22 & 20.24 & 58.48 & 27.30 & 29.87 & 24.10 & 21.12 & 24.38 & 23.98 \\
\hline 5 & 3-Pentanone, 2-methyl- & $\mathrm{C}_{6} \mathrm{H}_{12} \mathrm{O}$ & 5.23 & 755.70 & 752 & MS, RI & - & - & - & - & - & - & - & 1.88 & - & - & - \\
\hline 6 & 3-Hexen-2-one & $\mathrm{C}_{6} \mathrm{H}_{10} \mathrm{O}$ & 8.51 & 852.38 & 845 & MS, RI & - & - & - & - & 3.07 & 1.40 & 1.54 & 1.49 & 0.80 & 0.98 & 1.19 \\
\hline 7 & 2-Hexanone, 5-methyl- & $\mathrm{C}_{7} \mathrm{H}_{14} \mathrm{O}$ & 8.97 & 864.56 & 857 & MS, RI & - & - & - & 14.49 & - & - & - & - & - & - & - \\
\hline 8 & 2-Heptanone & $\mathrm{C}_{7} \mathrm{H}_{14} \mathrm{O}$ & 10.43 & 891.82 & 891 & MS, RI & 31.60 & 32.13 & 32.41 & 31.62 & 53.88 & 26.19 & 19.54 & 17.78 & 18.53 & 17.43 & 17.66 \\
\hline 9 & 3-Heptanone, 5-methyl- & $\mathrm{C}_{8} \mathrm{H}_{16} \mathrm{O}$ & 12.87 & 968.76 & 962 & MS, RI & 2.60 & 2.79 & 3.12 & 3.09 & - & - & - & - & - & - & - \\
\hline 10 & 2-Octanone & $\mathrm{C}_{8} \mathrm{H}_{16} \mathrm{O}$ & 14.15 & 1003.70 & 1003 & MS, RI & 14.44 & 15.14 & 15.44 & 15.34 & 17.96 & 9.16 & 7.58 & 7.34 & 7.55 & 7.19 & 7.22 \\
\hline 11 & 3-Octen-2-one & $\mathrm{C}_{8} \mathrm{H}_{14} \mathrm{O}$ & 15.79 & 1049.21 & 1046 & MS, RI & 7.55 & 7.83 & 7.85 & 7.52 & 9.88 & 5.00 & 4.98 & 4.53 & 1.18 & - & - \\
\hline 12 & 2-Nonanone & $\mathrm{C}_{9} \mathrm{H}_{18} \mathrm{O}$ & 17.54 & 1098.98 & 1096 & MS, RI & 26.03 & 28.38 & 32.78 & 28.02 & 26.66 & 13.78 & 7.39 & 5.95 & - & - & - \\
\hline 13 & 2-Undecanone & $\mathrm{C}_{11} \mathrm{H}_{22} \mathrm{O}$ & 23.52 & 1310.70 & 1305 & MS, RI & 3.41 & 3.48 & 3.89 & 3.33 & 3.90 & 2.18 & 1.78 & 1.45 & 1.70 & 1.10 & 1.43 \\
\hline \multicolumn{18}{|c|}{ Alcohols } \\
\hline 1 & Butanol, 3-methyl- & $\mathrm{C}_{5} \mathrm{H}_{12} \mathrm{O}$ & 5.71 & 772.37 & 767 & MS, RI & 5.17 & 4.28 & 5.03 & 18.39 & 23.37 & 10.80 & 10.76 & 10.83 & 10.39 & 11.29 & 11.09 \\
\hline 2 & 1-Pentanol & $\mathrm{C}_{5} \mathrm{H}_{12} \mathrm{O}$ & 5.71 & 772.37 & 772 & MS, RI & - & - & - & - & - & - & - & - & - & - & 10.95 \\
\hline 3 & 2-Heptanol, 3-methyl- & $\mathrm{C}_{8} \mathrm{H}_{18} \mathrm{O}$ & 7.23 & 818.65 & - & MS & - & - & - & - & 2.88 & 2.56 & 2.69 & 2.06 & - & - & - \\
\hline 4 & Hexanol & $\mathrm{C}_{6} \mathrm{H}_{14} \mathrm{O}$ & 9.66 & 882.39 & 880 & MS, RI & 40.75 & 42.70 & 49.69 & 53.72 & 152.25 & 74.39 & 44.01 & 38.35 & 43.49 & 43.49 & 41.13 \\
\hline 5 & Heptanol & $\mathrm{C}_{7} \mathrm{H}_{16} \mathrm{O}$ & 13.26 & 979.30 & 974 & MS, RI & 58.68 & 121.58 & 142.23 & 196.00 & 244.29 & 121.75 & 82.17 & 71.20 & 70.59 & 72.54 & 68.06 \\
\hline 6 & cis-Hept-4-enol & $\mathrm{C}_{7} \mathrm{H}_{14} \mathrm{O}$ & 13.26 & 979.36 & - & MS & - & 2.54 & 2.21 & 3.79 & 7.84 & 4.11 & 1.75 & 1.47 & 1.47 & 1.52 & 0.96 \\
\hline 7 & 1-Octen-3-ol & $\mathrm{C}_{8} \mathrm{H}_{16} \mathrm{O}$ & 13.75 & 991.78 & 986 & MS, RI & 4.93 & 7.35 & 4.47 & 5.78 & 9.88 & 4.16 & 2.21 & 1.66 & 1.10 & 1.87 & 1.09 \\
\hline 8 & 3,5-Octadien-2-ol & $\mathrm{C}_{8} \mathrm{H}_{14} \mathrm{O}$ & 15.32 & 1039.01 & 1037 & MS, RI & - & - & - & - & 10.36 & 6.66 & 1.67 & 1.78 & 1.88 & 1.89 & 1.19 \\
\hline 9 & 2-Octen-1-ol, (E)- & $\mathrm{C}_{8} \mathrm{H}_{16} \mathrm{O}$ & 15.41 & 1041.72 & 1059 & MS, RI & - & 3.21 & 2.81 & 1.87 & 1.69 & 1.65 & 0.73 & 0.74 & 0.57 & 0.64 & 0.34 \\
\hline 10 & 3,5-Octadien-2-ol & $\mathrm{C}_{8} \mathrm{H}_{14} \mathrm{O}$ & 15.80 & 1053.31 & 1039 & MS, RI & - & - & - & - & - & - & - & 1.67 & - & - & - \\
\hline 11 & 1-Octanol & $\mathrm{C}_{8} \mathrm{H}_{18} \mathrm{O}$ & 16.88 & 1086.07 & 1087 & MS, RI & - & 78.97 & 95.89 & - & - & - & - & - & - & - & - \\
\hline 12 & 5-Octen-2-ol, 5-methyl- & $\mathrm{C}_{9} \mathrm{H}_{18} \mathrm{O}$ & 17.54 & 1106.30 & - & MS & - & 30.29 & 24.30 & 19.03 & 17.98 & 17.88 & 14.98 & 15.08 & 13.68 & 9.62 & 9.80 \\
\hline 13 & 3-Decyn-2-ol & $\mathrm{C}_{10} \mathrm{H}_{18} \mathrm{O}$ & 17.70 & 1111.55 & 1101 & MS, RI & - & 3.05 & - & - & - & - & - & - & - & - & - \\
\hline 14 & 2-Nonen-1-ol, (E)- & $\mathrm{C}_{9} \mathrm{H}_{18} \mathrm{O}$ & 19.91 & 1183.74 & 1171 & MS, RI & - & - & - & - & 3.97 & 3.22 & 1.82 & 1.34 & 1.13 & 1.23 & 1.50 \\
\hline 15 & 1-Nonanol & $\mathrm{C}_{9} \mathrm{H}_{20} \mathrm{O}$ & 19.99 & 1186.58 & 1186 & MS, RI & - & 13.28 & 23.21 & 22.71 & 45.23 & 25.13 & 17.42 & 14.07 & 12.11 & 12.34 & 13.38 \\
\hline 16 & 1-Decanol & $\mathrm{C}_{10} \mathrm{H}_{22} \mathrm{O}$ & 22.90 & 1288.14 & 1279 & MS, RI & - & - & - & - & - & - & - & 1.96 & - & - & - \\
\hline \multicolumn{18}{|c|}{ Esters } \\
\hline 1 & Formic acid, hexyl ester & $\mathrm{C}_{7} \mathrm{H}_{14} \mathrm{O}_{2}$ & 11.87 & 941.92 & 927 & MS, RI & - & - & - & - & 2.54 & 2.09 & 2.66 & 1.98 & 1.07 & 1.27 & 1.01 \\
\hline 2 & Formic acid, octyl ester & $\mathrm{C}_{9} \mathrm{H}_{18} \mathrm{O}_{2}$ & 16.90 & 1086.66 & - & MS & - & - & 87.96 & 77.06 & 132.20 & 68.07 & 62.10 & 55.59 & 43.15 & 39.37 & 32.29 \\
\hline 3 & 1-Octen-3-yl-acetate & $\mathrm{C}_{10} \mathrm{H}_{18} \mathrm{O}_{2}$ & 17.25 & 1097.11 & 1097 & MS, RI & - & - & - & - & - & 1.20 & 1.09 & 1.34 & 1.32 & 1.02 & 1.21 \\
\hline 4 & Acetic acid, octyl ester & $\mathrm{C}_{10} \mathrm{H}_{20} \mathrm{O}_{2}$ & 20.34 & 1197.97 & 1200 & MS, RI & - & - & - & - & 7.83 & 3.29 & 2.44 & 2.09 & 1.79 & 1.23 & 1.24 \\
\hline 5 & Oxirane, decyl- & $\mathrm{C}_{12} \mathrm{H}_{24} \mathrm{O}$ & 23.51 & 1310.47 & 1307 & MS, RI & - & 2.43 & 2.48 & 2.07 & - & - & - & - & - & - & - \\
\hline
\end{tabular}


Table 1. Cont.

\begin{tabular}{|c|c|c|c|c|c|c|c|c|c|c|c|c|c|c|c|c|c|}
\hline & \multirow{2}{*}{ Volatile Compound } & \multirow{2}{*}{$\begin{array}{l}\text { Chemical } \\
\text { Formula }\end{array}$} & \multirow{2}{*}{$\mathrm{RT}^{1}$} & \multirow{2}{*}{$\mathrm{RI}^{2}$} & \multirow{2}{*}{$\mathbf{R I}^{3}$} & \multirow{2}{*}{ Method $^{4}$} & \multicolumn{11}{|c|}{$\mu \mathrm{g} / \mathrm{L}$} \\
\hline & & & & & & & $0 \mathrm{~h} \mathrm{(F)}{ }^{5}$ & $2 \mathrm{~h}(\mathrm{~F})$ & $4 \mathrm{~h} \mathrm{(F)}$ & $6 \mathrm{~h} \mathrm{(F)}$ & $0 \mathrm{~d}(\mathrm{~S})^{6}$ & 12 h (S) & $1 \mathrm{~d}(\mathrm{~S})$ & $2 \mathrm{~d}(\mathrm{~S})$ & $3 \mathrm{~d}(\mathrm{~S})$ & $7 \mathrm{~d}(\mathrm{~S})$ & $14 \mathrm{~d}(\mathrm{~S})$ \\
\hline \multicolumn{18}{|c|}{ Aromatic hydrocarbons } \\
\hline 1 & Cyclopentane, methyl- & $\mathrm{C}_{6} \mathrm{H}_{12}$ & 2.65 & 626.43 & 624 & MS, RI & - & 1.86 & 1.65 & - & - & - & - & - & - & - & - \\
\hline 2 & $n$-Hexane & $\mathrm{C}_{6} \mathrm{H}_{14}$ & 3.46 & 687.93 & - & MS & - & - & - & 2.52 & - & - & - & - & - & - & - \\
\hline 3 & Heptane & $\mathrm{C}_{7} \mathrm{H}_{16}$ & 3.58 & 697.48 & - & MS & - & - & - & - & 8.38 & 5.40 & 2.30 & 1.83 & 1.99 & 2.28 & 2.21 \\
\hline 4 & 1-Octene & $\mathrm{C}_{8} \mathrm{H}_{16}$ & 6.53 & 797.74 & 794 & MS, RI & 3.94 & 4.93 & 4.33 & 5.91 & - & - & - & - & - & - & - \\
\hline 5 & Hexane, 2,4-dimethyl- & $\mathrm{C}_{8} \mathrm{H}_{18}$ & 6.81 & 807.75 & - & MS & - & - & - & - & - & 15.65 & 7.43 & 7.78 & 1.62 & - & - \\
\hline 6 & 1-Nonene & $\mathrm{C}_{9} \mathrm{H}_{18}$ & 9.70 & 883.77 & 893 & MS, RI & 46.10 & 15.82 & 11.12 & 5.48 & - & - & - & - & - & - & - \\
\hline 7 & Octane, 2,7-dimethyl- & $\mathrm{C}_{10} \mathrm{H}_{22}$ & 11.55 & 933.18 & 934 & MS, RI & 3.33 & - & - & - & - & - & - & - & - & - & - \\
\hline 8 & 4,6-Decadiene & $\mathrm{C}_{10} \mathrm{H}_{18}$ & 18.53 & 1133.78 & 1145 & MS, RI & 4.02 & 3.96 & 4.23 & 4.29 & 4.33 & 3.02 & 2.78 & 1.40 & 1.40 & - & - \\
\hline 9 & 4-Dodecyne & $\mathrm{C}_{12} \mathrm{H}_{22}$ & 20.45 & 1201.69 & 1203.00 & MS, RI & - & - & - & 1.76 & - & - & - & - & - & - & - \\
\hline 10 & 3-Dodecyne & $\mathrm{C}_{12} \mathrm{H}_{22}$ & 20.78 & 1213.20 & 1210 & MS, RI & 3.37 & - & - & - & - & - & - & - & - & - & - \\
\hline 11 & 1-Octadecyne & $\mathrm{C}_{18} \mathrm{H}_{34}$ & 21.56 & 1240.88 & 1238 & MS, RI & 3.12 & 3.13 & - & - & - & - & - & - & - & - & - \\
\hline 12 & 5-Tridecene, (Z)- & $\mathrm{C}_{13} \mathrm{H}_{26}$ & 22.48 & 1273.35 & 1272.8 & MS, RI & 2.21 & 1.98 & 1.60 & 1.18 & 1.13 & 1.23 & 0.93 & 0.99 & 0.82 & 0.96 & 1.38 \\
\hline 13 & 2-Dodecenal, (E)- & $\mathrm{C}_{12} \mathrm{H}_{22} \mathrm{O}$ & 27.56 & 1457.11 & 1452 & MS, RI & - & 1.24 & 1.45 & 1.52 & 1.23 & 1.06 & 0.22 & - & - & - & - \\
\hline \multicolumn{18}{|c|}{ Internal standard } \\
\hline 1 & 1,2-Dichloro-benzene & $\mathrm{C}_{6} \mathrm{H}_{4} \mathrm{Cl}_{2}$ & 14.89 & 1018 & 1014 & MS, RI & 10 & 10 & 10 & 10 & 10 & 10 & 10 & 10 & 10 & 10 & 10 \\
\hline
\end{tabular}

${ }^{1} \mathrm{RT}$, retention time; ${ }^{2} \mathrm{RI}$, retention index. The RI of unknown compounds in an HP-5MS column calculated against the GC-MS retention time of $n$-alkanes (C3-C25); ${ }^{3} \mathrm{RI}$ from a database (http:/ / webbook.nist.gov / chemistry); ${ }^{4}$ RI, agreed with the retention index from the literature; MS, compared with NIST 11 Mass Spectral Database; STD, agreed with the mass spectrum of standard chemical; ' - ', not detected; ${ }^{5} \mathrm{~F}$, fermentation; ${ }^{6} \mathrm{~S}$, storage 


\subsection{Evaluation of $\mathrm{OAVS}$}

To determine how each volatile compound contributed to the overall odor profile of milk fermented by $S$. thermophilus MGA45-4, we calculated the OAVs for all samples during fermentation and storage (Table 2). Compounds with an OAV greater than one are considered to have a greater influence on the product's odor and flavor [20]. Among the 73 volatile compounds identified, some compounds were found to have a higher concentration than their detection threshold concentration (i.e., OAV > 1). Specifically, seven aldehyde compounds exhibited an OAV > 1, including octanal (4.21-127.93), nonanal (1.03-52.04), hexanal (1.32-33.03), (E)-2-octenal (1.16-6.83), 3-methyl-butanal (1.39-2.34), (E)-2-heptenal (0.23-1.81), and (E)-2-pentenal (0.85-1.61). This indicated that aldehydes are important contributors to the flavor of milk fermented by S. thermophilus MGA45-4. Of the 16 ketone compounds identified, only 2,3-butanedione (0.90-1.65) and acetoin (0.06-1.06) had an $\mathrm{OAV}>1$. Given that 2,3-butanedione is a well-known metabolite of LAB, it is likely that it makes a significant contribution to the good odor/flavor of milk fermented by S. thermophilus MGA45-4 [40]. Although alcohol compounds represent the largest group of volatile compounds identified in our study, only hexanol and 1-octen-3-ol had an OAV greater than one, suggesting that they may play a role in the odor/flavor of related dairy products [6]. This result is consistent with Attaie [41].

Almost all of the acid compounds that had a higher reported threshold value had low concentrations in the milk fermented by S. thermophilus MGA45-4. For example, the threshold value of acetic acid was 32,300 $\mu \mathrm{g} / \mathrm{L}$ and the OAV was $<1$ (0.00053-0.00217). Similar acid compounds were hexanoic acid (threshold value, 27,100 $\mu \mathrm{g} / \mathrm{L}$ ) and octanoic acid (threshold value, 11,300 $\mu \mathrm{g} / \mathrm{L}$ ) [42].

The physicochemical characteristics of fermented milk can affect the release of volatile compounds from the microstructure of food matrices. Most of the key flavor compounds (OAV >1) were detected on day 0 of storage. This result also indicated that the flavor on day 0 of storage was better than at other time points. On day 0 of storage, although the $\mathrm{pH}$ of the samples decreased to 4.5, the TA increased to $75.72{ }^{\circ} \mathrm{T}$, and the viable cell count increased to $8.99 \log _{10} \mathrm{CFU} / \mathrm{mL}$. These changes in the physicochemical characteristics enable the release of flavored compounds. The results of sensory assessment are consistent with this conclusion. 
Table 2. Odor activity values (OAVs) for compounds produced in milk fermented by S. thermophilus MGA45-4 during fermentation and storage.

\begin{tabular}{|c|c|c|c|c|c|c|c|c|c|c|c|c|c|c|}
\hline \multirow{2}{*}{ Volatile Compound } & \multirow{2}{*}{$\begin{array}{l}\text { Chemical } \\
\text { Formula }\end{array}$} & \multirow{2}{*}{$\begin{array}{l}\text { Odor threshold } \\
(\mu \mathrm{g} / \mathrm{L})\end{array}$} & \multirow{2}{*}{ References } & \multicolumn{11}{|c|}{ OAV } \\
\hline & & & & $0 \mathrm{~h}(\mathrm{~F})^{1}$ & $2 \mathrm{~h} \mathrm{(F)}$ & $4 \mathrm{~h} \mathrm{(F)}$ & $6 \mathrm{~h}(\mathrm{~F})$ & $0 \mathrm{~d}(\mathrm{~S})^{2}$ & $12 \mathrm{~h} \mathrm{(S)}$ & $1 \mathrm{~d}(\mathrm{~S})$ & $2 \mathrm{~d}(\mathrm{~S})$ & $3 \mathrm{~d}(\mathrm{~S})$ & $7 \mathrm{~d}(\mathrm{~S})$ & $14 \mathrm{~d}(\mathrm{~S})$ \\
\hline Butanal, 3-methyl- & $\mathrm{C}_{5} \mathrm{H}_{10} \mathrm{O}$ & 5.4 & [42] & 1.39 & 2.78 & 3.14 & 2.34 & - & - & - & - & - & - & - \\
\hline 2-Pentenal, (E)- & $\mathrm{C}_{5} \mathrm{H}_{8} \mathrm{O}$ & 1.2 & [38] & - & - & - & 1.61 & 0.96 & 0.86 & 0.85 & 1.03 & 1.04 & 1.33 & 1.57 \\
\hline Hexanal & $\mathrm{C}_{6} \mathrm{H}_{12} \mathrm{O}$ & 3 & [38] & 33.03 & 30.53 & 19.37 & 17.34 & 1.32 & - & - & - & - & - & - \\
\hline 2-Heptenal, (E)- & $\mathrm{C}_{7} \mathrm{H}_{12} \mathrm{O}$ & 13 & [43] & 0.77 & 0.88 & 0.80 & 0.89 & 1.81 & 0.57 & 0.26 & 0.48 & 0.23 & 0.38 & 0.44 \\
\hline Octanal & $\mathrm{C}_{8} \mathrm{H}_{16} \mathrm{O}$ & 0.7 & [38] & 127.93 & 64.46 & 33.56 & 26.06 & 11.04 & 4.21 & - & - & - & - & - \\
\hline 2-Octenal, (E)- & $\mathrm{C}_{8} \mathrm{H}_{14} \mathrm{O}$ & 3 & [44] & 4.43 & 4.62 & 5.02 & 5.22 & 6.83 & 3.40 & 1.58 & 1.48 & 1.19 & 1.16 & 1.43 \\
\hline Nonanal & $\mathrm{C}_{9} \mathrm{H}_{18} \mathrm{O}$ & 1 & [45] & 52.04 & 35.95 & 28.60 & 9.58 & 4.01 & 1.52 & 1.43 & 1.03 & 1.50 & 1.12 & 1.13 \\
\hline 2,3-Butanedione & $\mathrm{C}_{4} \mathrm{H}_{6} \mathrm{O}_{2}$ & 10 & [42] & - & - & - & - & 1.61 & 1.65 & 1.53 & 1.61 & 0.90 & - & - \\
\hline Acetoin & $\mathrm{C}_{4} \mathrm{H}_{8} \mathrm{O}_{2}$ & 55 & [44] & - & - & 0.06 & 0.37 & 1.06 & 0.50 & 0.54 & 0.44 & 0.38 & 0.44 & 0.44 \\
\hline Hexanol & $\mathrm{C}_{6} \mathrm{H}_{14} \mathrm{O}$ & 120 & [42] & 0.34 & 0.36 & 0.41 & 0.45 & 1.27 & 0.62 & 0.37 & 0.32 & 0.36 & 0.36 & 0.34 \\
\hline 1-Octen-3-ol & $\mathrm{C}_{8} \mathrm{H}_{16} \mathrm{O}$ & 10 & [23] & 0.49 & 0.74 & 0.45 & 0.58 & 0.99 & 0.42 & 0.22 & 0.17 & 0.11 & 0.19 & 0.11 \\
\hline
\end{tabular}




\section{Experimental}

\subsection{Bacterial Isolates and Reagents}

Streptococcus thermophilus MGA45-4 was originally isolated from traditional fermented milk collected in Kent Province, Mongolia, and was used throughout this study [22]. Standard $n$-alkanes $\left(C_{3}-C_{25}\right)$ were obtained from AccuStandard (New Haven, CT, USA). 1,2-Dichloro-benzene was used as an internal standard (ISTD) and was purchased from Sigma-Aldrich (Steinheim, Germany). MRS (De Man, Rogosa and Sharpe) broth was purchased from OXOID (Hampshire, England) and whole milk powder was purchased from NZMP (Wellington, New Zealand).

\subsection{Preparation of Fermented Milk}

Fermented milk was prepared using a previously described method [40] with some modifications. Briefly, frozen $S$. thermophilus MGA45-4 cells were propagated routinely by culturing in MRS broth for $24 \mathrm{~h}$ at $37^{\circ} \mathrm{C}$ at least three times, followed by inoculation in milk/sucrose medium. Sterile milk was prepared by reconstituting $11.5 \%(w / v)$ whole milk powder in distilled water and heating to $50{ }^{\circ} \mathrm{C}$ for approximately $30 \mathrm{~min}$, followed by supplementation with $6.5 \mathrm{~g} / 100 \mathrm{~g}$ of sucrose. The resultant medium was sterilized by heating at $95{ }^{\circ} \mathrm{C}$ for $5 \mathrm{~min}$ and stored at $4{ }^{\circ} \mathrm{C}$ before use. S. thermophilus MGA45-4 was inoculated at a concentration of $5 \times 10^{7}$ colony-forming units (CFU)/mL. After the milk coagulated, samples were incubated at $42{ }^{\circ} \mathrm{C}$ until the $\mathrm{pH}$ dropped to 4.5 and were then stored at $4{ }^{\circ} \mathrm{C}$ for 14 days. Samples were taken from each culture after $0 \mathrm{~h}, 2 \mathrm{~h}, 4 \mathrm{~h}$, and $6 \mathrm{~h}$ of fermentation and $0 \mathrm{~d}, 12 \mathrm{~h}, 1 \mathrm{~d}, 2 \mathrm{~d}, 3 \mathrm{~d}, 7 \mathrm{~d}$, and $14 \mathrm{~d}$ of storage. The fermented milk samples were frozen at $-20^{\circ} \mathrm{C}$ before analysis.

\subsection{Determining Viable Cell Counts}

Viable S. thermophilus MGA45-4 cells were counted using the pour-plate method, as previously described [46]. To enumerate viable cells, a $1 \mathrm{~mL}$ sample was diluted in $9 \mathrm{~mL}$ of sterile physiological saline $(0.85 \%, w / v)$ and suitable dilutions were plated on MRS agar. Plates were incubated under anaerobic conditions at $42{ }^{\circ} \mathrm{C}$ for $48 \mathrm{~h}$. Colonies were counted on each plate and viable cell counts were expressed as $\log _{10} \mathrm{CFU} / \mathrm{mL}$.

\subsection{Determination of $\mathrm{pH}$ and $\mathrm{TA}$}

The $\mathrm{pH}$ was measured at $20^{\circ} \mathrm{C}$ using a pHS-3C precision $\mathrm{pH}$ meter (Leici Devices, Shanghai, China). TA was determined as previously described [47]. Five-gram samples were weighed and mixed with $40 \mathrm{~mL}$ of water and then titrated with standardized $0.1 \mathrm{~N} \mathrm{NaOH}$ to a pH of 8.1 using $0.5 \%$ phenolphthalein as an indicator.

\subsection{Determination of Viscosity}

Viscosity was measured using a Brookfield DV-E Viscometer (Brookfield Engineering Laboratories, Middleboro, MA, USA). All samples were spun at $100 \mathrm{rpm}$ for $30 \mathrm{~s}$ using a Brookfield DV-1 viscometer with a No. 4 spindle [48]. The viscosity was expressed in $\mathrm{mPa} \cdot \mathrm{s}$.

\subsection{Determination of WHC}

The WHC is defined as the ability of fermented milk to hold all or part of its own water [49]. Fermented milk ( $20 \mathrm{~g}$ ) was placed in a funnel containing filter paper and allowed to run through at $21{ }^{\circ} \mathrm{C}$ for $120 \mathrm{~min}$. The filtrate was collected and weighed. The WHC was calculated using the following equation:

$$
\mathrm{WHC}(\%)=(1-\mathrm{W} 1 / \mathrm{W} 2) \times 100 \% \text {, }
$$

where W1 = weight of filtrate in grams; and W2 = weight of fermented milk in grams. 


\subsection{HS-SPME-GC-MS Analysis}

An SPME fiber (50/30 $\mu \mathrm{m}$ divinylbenzene/Carboxen/polydimethylsiloxane; Supelco, Bellefonte, PA, USA) was tested and used to identify the volatile compounds produced by $S$. thermophilus MGA45-4 in the fermented milk samples according to described methods [16]. The fiber was exposed for $60 \mathrm{~min}$ in the headspace of $20 \mathrm{~mL}$ glass vials (CNW Technologies, Germany) fitted with a polytetrafluoroethylene/silicone septum. Each glass vial contained $5 \mathrm{~mL}$ of sample and $10 \mu \mathrm{g} / \mathrm{L}$ of the ISTD. Subsequently, the fiber was immediately placed in the injection port of a 7890B GC system (Agilent Technologies, Palo Alto, CA, USA) for $5 \mathrm{~min}$ at $270{ }^{\circ} \mathrm{C}$ to allow for desorption of the volatile compounds into the GC.

\subsection{Identification of Volatile Compounds}

Absorbed volatiles were analyzed using a 7890 B GC equipped with a 5977 A mass selective detector (MSD; Agilent Technologies) and an HP-5MS column (30-m length, 0.25-mm i.d., 0.25- $\mu \mathrm{m}$ film thickness; Agilent Technologies). Helium was used as the carrier gas at $1 \mathrm{~mL} / \mathrm{min}$. The oven temperature was set at $35^{\circ} \mathrm{C}$ for $5 \mathrm{~min}$, increased to $140{ }^{\circ} \mathrm{C}$ at a rate of $4{ }^{\circ} \mathrm{C} / \mathrm{min}$ for $5 \mathrm{~min}$, and then increased further to $250{ }^{\circ} \mathrm{C}$ at a rate of $10^{\circ} \mathrm{C} / \mathrm{min}$. A final 5 -min extension was performed at $250{ }^{\circ} \mathrm{C}$. The ion source and the transfer line temperatures were set at $230{ }^{\circ} \mathrm{C}$ and $250^{\circ} \mathrm{C}$, respectively. The mass spectra of the samples were recorded with a scan range of 40-400 m/z and electronic impact (EI) mode at $70 \mathrm{eV}$. n-Alkanes (C3-C25) were used under the same experimental conditions to calculate the RIs of the volatile aroma compounds in each sample. All volatile compounds were semi-quantified and the results are shown as the retention time and relative peak area.

\subsection{Determination of $O A V$}

The OAV refers to the ratio of the concentration of a compound to its detection threshold concentration [19]. The OAVs in this study were calculated as previously described [20].

\subsection{Sensory Evaluation}

The flavors of the fermented milk products were assessed by six trained panelists. A beaker (100 mL) filled with the sample was used for evaluation. Flavor intensity was recorded on a scale ranging from 1 (strongly attractive) to 5 (strongly unappealing), according to international standards. Water was provided for mouth washing between samples.

\subsection{Statistical Analysis}

The data were analyzed with ANOVA using Proc Mixed (SAS Institute, Cary NC, USA). Significant differences between means were determined using Fisher's protected least significant difference test. Significant differences were defined at $p<0.05$ (SAS Institute, 1998). All measurements were performed in triplicate.

\section{Conclusions}

In this study, we characterized the physiochemical properties of milk fermented by $S$. thermophilus MGA45-4 by monitoring the viable cell count, $\mathrm{pH}$, TA, viscosity, and WHC of milk samples during fermentation and storage at $4{ }^{\circ} \mathrm{C}$. Our data indicate that the product had a viable count of $>8.86 \log _{10}$ $\mathrm{CFU} / \mathrm{mL}$ during both the fermentation and storage stages. A pH of 4.4 and viscosity of $834.33 \mathrm{mPa} \cdot \mathrm{s}$ were maintained in the storage stage. By analyzing the volatile compound profile of fermented milk using SPME-GC-MS, we identified 73 volatile compounds including 5 carboxylic acids, 21 aldehydes, 13 ketones, 16 alcohols, 5 esters, and 13 aromatic carbohydrates from samples undergoing fermentation and during storage. As indicated by their OAVs, some compounds were found to be the key factors determining the product odor/flavor. In particular, octanal, nonanal, hexanal, 2,3-butanedione, and 1-octen-3-ol, which had the highest OAVs among all compounds analyzed, likely contributed 
the most to the characteristic flavor of fermented milk. Together, our study provides an additional chemical/molecular basis for a better understanding of the aroma characteristics of fermented milk. This in turn may help improve the flavor quality of dairy products during the process of fermentation and storage.

Acknowledgments: This research was supported by the National Natural Science Foundation of China (Beijing; No. 31460446, 31471711).

Author Contributions: T.D. and T.S. conceived and designed the experiments; R.J. and W.R. performed the experiments; T.L. analyzed the data; H.C. contributed reagents/materials/analysis tools; T.D. and T.S. wrote the paper.

Conflicts of Interest: The authors declare no conflicts of interest.

\section{References}

1. Del, R.B.; Linares, D.M.; Ladero, V.; Redruello, B.; Fernández, M.; Martin, M.C.; Alvarez, M.A. Putrescine production via the agmatine deiminase pathway increases the growth of Lactococcus.Lactococcus lactis and causes the alkalinization of the culture medium. Appl. Microbiol. Blot. 2015, 99, 897-905. [CrossRef] [PubMed]

2. Blaiotta, G.; Sorrentino, A.; Ottombrino, A.; Aponte, M. Short communication: technological and genotypic comparison between Streptococcus macedonicus and Streptococcus thermophilus strains coming from the same dairy environment. J. Dairy Sci. 2011, 94, 5871-5877. [CrossRef] [PubMed]

3. Fatih, O.; Broadbent, J.R.; Oberg, C.J.; Mcmahon, D.J. Growth and gas formation by Lactobacillus wasatchensis, a novel obligatory heterofermentative nonstarter lactic acid bacterium, in Cheddar-style cheese made using a Streptococcus thermophilus starter. J. Dairy Sci. 2015, 98, 7473-7482.

4. Han, N.; Park, S.Y.; Kim, S.Y.; Yoo, M.Y.; Paik, H.D.; Lim, S.D. Short communication: Change of naturally occurring benzoic acid during skim milk fermentation by commercial cheese starters. J. Dairy Sci. 2016, 99, 8633-8637. [CrossRef] [PubMed]

5. Li, C.; Song, J.; Kwok, L.Y.; Wang, J.; Dong, Y.; Yu, H.; Hou, Q.; Zhang, H.; Chen, Y. Influence of Lactobacillus plantarum on yogurt fermentation properties and subsequent changes during postfermentation storage. J. Dairy Sci. 2017, 100, 2512-2525. [CrossRef] [PubMed]

6. Ning, L.; Fu-Ping, Z.; Hai-Tao, C.; Si-Yuan, L.; Chen, G.; Zhen-Yang, S.; Bao-Guo, S. Identification of volatile components in Chinese Sinkiang fermented camel milk using SAFE, SDE, and HS-SPME-GC/MS. Food Chem. 2011, 129, 1242-1252. [CrossRef] [PubMed]

7. Leroy, F.; Vuyst, L.D. Lactic acid bacteria as functional starter cultures for the food fermentation industry. Trends Food Sci. Technol. 2004, 15, 67-78. [CrossRef]

8. Condurso, C.; Verzera, A.; Romeo, V.; Ziino, M.; Conte, F. Solid-phase microextraction and gas chromatography mass spectrometry analysis of dairy product volatiles for the determination of shelf-life. Int. Dairy J. 2008, 18, 819-825. [CrossRef]

9. Pan, D.D.; Wu, Z.; Peng, T.; Zeng, X.Q.; Li, H. Volatile organic compounds profile during milk fermentation by Lactobacillus pentosus and correlations between volatiles flavor and carbohydrate metabolism. J. Dairy Sci. 2014, 97, 624-631. [CrossRef] [PubMed]

10. Settachaimongkon, S.; Nout, M.J.; Antunes Fernandes, E.C.; Hettinga, K.A.; Vervoort, J.M.; van Hooijdonk, T.C.; Zwietering, M.H.; Smid, E.J.; van Valenberg, H.J. Influence of different proteolytic strains of Streptococcus thermophilus in co-culture with Lactobacillus delbrueckii subsp. bulgaricus on the metabolite profile of set-yoghurt. Int. J. Food Microbiol. 2014, 177, 29-36. [CrossRef] [PubMed]

11. Ziadi, M.; Wathelet, J.P.; Marlier, M.; Hamdi, M.; Thonart, P. Analysis of volatile compounds produced by 2 strains of lactis isolated from leben (Tunisian fermented milk) using solid-phase microextraction-gas chromatography. J. Food Sci. 2008, 73, 247-252. [CrossRef]

12. Beshkova, D.M.; Simova, E.D.; Frengova, G.I.; Simov, Z.I.; Dimitrov, Z.P. Production of volatile aroma compounds by kefir starter cultures. Int. Dairy J. 2003, 13, 529-535. [CrossRef]

13. Hols, P.; Hancy, F.; Fontaine, L.; Grossiord, B.; Prozzi, D.; Leblond-Bourget, N.; Decaris, B.; Bolotin, A.; Delorme, C.; Dusko, E.S. New insights in the molecular biology and physiology of Streptococcus thermophilus revealed by comparative genomics. Fems. Microbiol. Rev. 2005, 29, 435-463. [PubMed] 
14. Linares, D.M.; O'Callaghan, T.F.; O'Connor, P.M.; Ross, R.P.; Stanton, C. Streptococcus thermophilus APC151 strain is suitable for the manufacture of naturally GABA-enriched bioactive yogurt. Front. Microbiol. 2016, 7, 1876. [CrossRef] [PubMed]

15. Rabha, B.; Nadra, R.S.; Ahmed, B. Effect of threonine on growth and acetaldehyde production by streptococcus thermophilus. World Appl. Sci. J. 2011, 15, 160-163.

16. Dan, T.; Wang, D.; Jin, R.L.; Zhang, H.P.; Zhou, T.T.; Sun, T.S. Characterization of volatile compounds in fermented milk using solid-phase microextraction methods coupled with gas chromatography-mass spectrometry. J. Dairy Sci. 2017, 100, 2488-2500. [CrossRef] [PubMed]

17. Ferreira, V.; Ortín, N.; Escudero, A.; López, R.; Cacho, J. Chemical characterization of the aroma of Grenache rose wines: aroma extract dilution analysis, quantitative determination, and sensory reconstitution studies. J. Agric. Food Chem. 2002, 50, 4048. [CrossRef] [PubMed]

18. Capone, S.; Tufariello, M.; Siciliano, P. Analytical characterisation of Negroamaro red wines by "Aroma Wheels". Food Chem. 2013, 141, 2906-2915. [CrossRef] [PubMed]

19. González, Á.M.; Gonzálezbarreiro, C.; Canchogrande, B.; Simalgándara, J. Relationships between Godello white wine sensory properties and its aromatic fingerprinting obtained by GC-MS. Food Chem. 2011, 129, 890-898. [CrossRef] [PubMed]

20. Wang, D.; Dan, T.; Sun, T.; Zhang, H.; Menghe, B. Analysis of key volatile compounds in fermented cow milk produced by pure and mixed cultures by SPME-GC-MS combined with ROAV. Food Sci. 2017, 38, 145-152.

21. Ayad, E.H.E.; Verheul, A.; Wouters, J.T.M.; Smit, G. Application of wild starter cultures for flavour development in pilot plant cheese making. Int. Dairy J. 2000, 10, 169-179. [CrossRef]

22. Ren, Y.; Liu, W.; Zhang, H. Identification of coccoidal bacteria in traditional fermented milk products from Mongolia, and the fermentation properties of the predominant species, Streptococcus thermophilus. Korean J. Food Sci. Anim. Resour. 2015, 35, 683-691. [CrossRef] [PubMed]

23. Molimard, P.; Spinnler, H.E. Review: Compounds Involved in the Flavor of Surface Mold-Ripened Cheeses: Origins and Properties. J. Dairy Sci. 1996, 79, 169-184. [CrossRef]

24. Cheng, H. Volatile flavor compounds in yogurt: a review. Crit. Rev. Food Sci. 2010, 50, 938-950. [CrossRef] [PubMed]

25. Panagiotidis, P.; Tzia, C.; Spanier, A.M.; Shahidi, F.; Parliament, T.H.; Mussinan, C.; Ho, C.T.; Contis, E.T. Effect of milk composition and heating on flavor and aroma of yogurt. SPEC Publ.-R. Soc. Chem. 2001, 274, 160-170.

26. Patton, S. Flavor thresholds of volatile fatty acids. J. Food Sci. 2010, 29, 679-680. [CrossRef]

27. Afzal, M.I.; Delaunay, S.; Paris, C.; Borges, F.; Revol-Junelles, A.M.; Cailliez-Grimal, C. Identification of metabolic pathways involved in the biosynthesis of flavor compound 3-methylbutanal from leucine catabolism by Carnobacterium.Carnobacterium maltaromaticum LMA 28. Int. J. Food Microbiol. 2012, 157, 332-339. [CrossRef] [PubMed]

28. Afzal, M.I.; Boulahya, K.A.; Jacquot, M.; Delaunay, S.; Cailliezgrimal, C. Biosynthesis and role of 3-methylbutanal in cheese by lactic acid bacteria: Major metabolic pathways, enzymes involved, and strategies for control. Crit. Rev. Food Sci. 2015, 57, 339. [CrossRef] [PubMed]

29. Peralta, G.H.; Wolf, I.V.; Bergamini, C.V.; Perotti, M.C.; Hynes, E.R. Evaluation of volatile compounds produced by Lactobacillus paracasei 190 in a hard-cooked cheese model using solid-phase microextraction. Dairy Sci. Technol. 2014, 94, 73-81. [CrossRef]

30. Chu, F.L.; Yaylayan, V.A. Model studies on the oxygen-induced formation of benzaldehyde from phenylacetaldehyde using pyrolysis gc-ms and ftir. Agric. Food Chem. 2008, 56, 10697-10704. [CrossRef] [PubMed]

31. Sidira, M.; Kandylis, P.; Kanellaki, M.; Kourkoutas, Y. Effect of immobilized Lactobacillus casei on the evolution of flavor compounds in probiotic dry-fermented sausages during ripening. Meat Sci. 2015, 100, 41-51. [CrossRef] [PubMed]

32. Marco, A.; Navarro, J.L.; Flores, M. The influence of nitrite and nitrate on microbial, chemical and sensory parameters of slow dry fermented sausage. Meat Sci. 2006, 73, 660. [CrossRef] [PubMed]

33. Jensen, K.A.; Evans, K.M.; Kirk, T.K.; Hammel, K.E. Biosynthetic pathway for veratryl alcohol in the ligninolytic fungus phanerochaete chrysosporium. Appl. Environ. Microb. 1994, 60, 709-714.

34. Adhikari, K.; Hein, K.A.; Elmore, J.R.; Heymann, H.; Willott, A.M. Flavor threshold as affected by interaction among three dairy-related flavor compounds. J. Sens. Stud. 2010, 21, 626-643. [CrossRef] 
35. Hayaloglu, A.A.; Karabulut, I. SPME/GC-MS characterization and comparison of volatiles of eleven varieties of turkish cheeses. Int. J. Food Prop. 2013, 16, 1630-1653. [CrossRef]

36. Smit, G.; Smit, B.A.; Engels, W.J. Flavour formation by lactic acid bacteria and biochemical flavour profiling of cheese products. Fems. Microbiol. Rev. 2005, 29, 591-610. [CrossRef] [PubMed]

37. Rincon-Delgadillo, M.I.; Lopez-Hernandez, A.; Wijaya, I.; Rankin, S.A. Diacetyl levels and volatile profiles of commercial starter distillates and selected dairy foods. J. Dairy Sci. 2012, 95, 1128. [CrossRef] [PubMed]

38. Sun, B.G. Flavoring; Chemical Industry Press: Beijing, China, 2003; pp. 20-28.

39. Angerosa, F.; Camera, L.; d'Alessandro, A.N.; Mellerio, G. Characterization of seven new hydrocarbon compounds present in the aroma of virgin olive oils. J. Agric. Food Chem. 1998, 46, 648. [CrossRef] [PubMed]

40. Zha, M.; Yu, J.; Zhang, Y.; Wang, H.; Bai, N.; Qin, Y.; Liangliang, D.; Liu, W.; Zhang, H.; Bilige, M. Study on Streptococcus thermophilus isolated from Qula and associated characteristic of acetaldehyde and diacetyl in their fermented milk. J. Gen. Appl. Microbiol. 2015, 61, 50-56. [CrossRef] [PubMed]

41. Attaie, R. Quantification of volatile compounds in goat milk Jack cheese using static headspace gas chromatography. J. Dairy Sci. 2009, 92, 2435. [CrossRef] [PubMed]

42. Qian, M.; Reineccius, G.A. Quantification of aroma compounds in parmigiano reggiano cheese by a dynamic headspace gas chromatography-mass spectrometry technique and calculation of odor activity value. J. Dairy Sci. 2003, 86, 770-776. [CrossRef]

43. Leffingwell, J.C.; Leffingwell, D. GRAS flavor chemicals-detection thresholds. Perfumer Flavorist. 1991, 16, 2-19.

44. John, C. Flavor-Base (Demo), 10th ed.; Leffingwell and Associates: Canton, GA, USA, 2001.

45. Gemert, L.J.V. Compilations of Odour Threshold Values in Air, Water and Other Media, 10th ed.; Oliemans Punter \& Partners: Utrecht, The Netherlands, 2003.

46. Tharmaraj, N.; Shah, N.P. Selective enumeration of Lactobacillus delbrueckii ssp. bulgaricus, Streptococcus thermophilus, Lactobacillus acidophilus, Bifidobacteria, Lactobacillus casei, Lactobacillus rhamnosus, and Propionibacteria. J. Dairy Sci. 2003, 86, 2288-2296. [CrossRef]

47. Bao, Y.; Zhang, Y.; Li, H.; Liu, Y.; Wang, S.; Dong, X.; Su, F.; Yao, G.; Sun, T.; Zhang, H. In vitro screen of Lactobacillus plantarum as probiotic bacteria and their fermented characteristics in soymilk. Ann. Microbiol. 2012, 62, 1311-1320. [CrossRef]

48. Galmarini, M.V.; Baeza, R.; Sanchez, V.; Zamora, M.C.; Chirife, J. Comparison of the viscosity of trehalose and sucrose solutions at various temperatures: Effect of guar gum addition. LWT-Food Sci. Technol. 2011, 44, 186-190. [CrossRef]

49. Li, C.; Li, W.; Chen, X.; Feng, M.; Rui, X.; Jiang, M.; Dong, M. Microbiological, physicochemical and rheological properties of fermented soymilk produced with exopolysaccharide (EPS) producing lactic acid bacteria strains. LWT Food Sci. Technol. 2014, 57, 477-485. [CrossRef]

Sample Availability: Not available.

(C) 2018 by the authors. Licensee MDPI, Basel, Switzerland. This article is an open access article distributed under the terms and conditions of the Creative Commons Attribution (CC BY) license (http:/ / creativecommons.org/licenses/by/4.0/). 